\title{
Temperature Effects in ESR Spectra of Radical Centres in Dripstone Calcite Samples Used for ESR Dating
}

\author{
M. Wencka ${ }^{a, *}$, S.K. Hoffmann ${ }^{a}$, R. Krzyminiewski ${ }^{b}$ \\ AND S. MIELCAREK ${ }^{b}$ \\ ${ }^{a}$ Institute of Molecular Physics, Polish Academy of Sciences \\ Smoluchowskiego 17, 60-179 Poznań, Poland \\ ${ }^{b}$ Institute of Physics, Adam Mickiewicz University \\ Umultowska 85, 61-614 Poznań, Poland
}

(Received January 31, 2005; in final form April 20, 2005)

\begin{abstract}
Natural calcite samples were collected from the dripstone layers of the Black Cave of the Tatra Mountains. ESR spectra, thermal analysis, and thermoluminescence data were analysed to obtain a consistent picture of the calcite properties. The ESR spectra were analysed using computer enhanced resolution spectra and an influence of the thermal annealing on intensity of individual ESR lines was measured. These results together with the effects of $\gamma$-irradiation in ESR spectra allowed one to discriminate between ESR lines from various paramagnetic centres. A general conclusion of the paper is that in the ESR dating the outer ESR lines of $\mathrm{CO}_{3}^{-}(g=2.0125)$ and $\mathrm{CO}_{2}^{-}(g=1.9980)$ radicals should be used instead of the commonly used multicomponent central line.
\end{abstract}

PACS numbers: 76.30.- -v, 65.90.+i

\section{Introduction}

Electron spin resonance (ESR) dating in geochronology, dendrochronology, and archaeometry is a well-known method complementary to the ${ }^{14} \mathrm{C}$-dating $[1,2]$. The ESR method spans the time interval between old limit of ${ }^{14} \mathrm{C}$ (50 kyears) and young limit of the $\mathrm{K}$-Ar-dating (100 kyears) and allows dating up to about 1 million years. Routine procedures for the ESR dating are still not established although it is well recognized that sample preparation, measurement conditions,

*corresponding author; e-mail: mwencka@ifmpan.poznan.pl 
selection of appropriate ESR lines, and accumulated radiation dose determination using ESR line intensity can have an essential influence on a final result $[1,3,4]$. Especially important are chemical, mechanical, and thermal pre-treatments of a sample.

In geochronology and archaeometry calcite is the most intensively used material for ESR dating. In this paper we present temperature effects in ESR spectra of various paramagnetic centres in natural calcite (speleothems). Variations of temperature or annealing of samples prior to measurements can reduce a number of lines in ESR spectrum or can influence relative amplitudes of the lines by eliminating some unstable radical centres or by broadening the lines. The thermal treatment differentiates between individual line behaviour allowing an identification various paramagnetic centres and facilitates a choice of ESR line appropriate for the dating.

Paramagnetic centres in geological and archaeological materials are produced by natural $\alpha, \beta, \gamma$ irradiations resulting from decay of uranium and thorium nuclei and by $\beta$ irradiation from potassium nuclei, with a typical average annual dose order of 0.1-10 mGy. The expected radiation defects are of various types of carbonate radicals $\left(\mathrm{CO}_{3}^{3-}, \mathrm{CO}_{3}^{3-}\right.$, and $\left.\mathrm{CO}_{2}^{-}\right)$and trapped electron (colour centres). In fact such intrinsic centres were identified in pure synthetic $\mathrm{CaCO}_{3}$ after irradiation and their ESR parameters were determined from single crystal measurements $[1,5,6]$. Also ESR spectra of intentionally doped impurities in synthetic calcite were measured [7-9].

Natural calcite contains, usually, various types of impurities as admixtures of other carbonates, inorganic salts, and organic compounds. Cave deposits called dripstones or speleothems (stalactites, stalagmites), like the ones studied in this paper, appear as a result of the karst formation that is a dissolving of carbonate rocks by water containing $\mathrm{CO}_{2}$ from the air or from the soil. After entering a cave from the ceiling the migrating solution releases the gaseous carbon dioxide and the crystallization of a calcite dripstone occurs. Intensity of the karst formation process depends on the concentration of the acidic calcium carbonate in the water solution, the amount of the solution and its flow rate, and on temperature and partial pressure of $\mathrm{CO}_{2}[10,11]$. The migrating water contains many impurities from inorganic materials of the penetrated rocks and clay minerals, and organic materials dissolved during migration through the soil. The impurities can precipitate in the crystallising calcite in the form of inclusions or individual ionic or molecular impurities substituting host ion sites. Many humic and non-humic organic substances have been recognized in cave calcite deposits. The specific humus substances include fulvic and humic acids, whereas the non-specific substances are: carbohydrates, lignin, proteins, tanning substances, waxes, and tars with contents of the organic carbon in humus up to $70 \%[12,13]$. A concentration of humus in the solution from which dripstones crystallise depends on the rate of washing out the organic matter from the soil, vegetation cover, rate of flow of rinsing water 
and the distance from the soil surface to the dripstone crystallisation site. The greatest amount of organic substances accumulate in a dripstone during spring or autumn and the smallest one - during summer seasons.

All the impurities existing in natural calcite can contain various paramagnetic centres or the centres can be induced during artificial irradiation. An identification of types and concentration of paramagnetic defects by ESR for a given speleothem gives a picture characteristic of the cave and characterises also its geological history. Superposition of ESR lines from various impurities in an ESR spectrum makes the interpretation far from trivial.

Dating of cave deposits by ESR can be interfered by thermal history of a sample (hot springs, lava) which can affect paramagnetic centres in various manners. Thus important seems to be the knowledge how the temperature or sample annealing influences concentration and dynamic properties of the centres used in the ESR dating. In this paper we describe results of thermal studies of dripstone calcite by thermal analysis methods (derivative thermogravimetry analysis (DTA), thermogravimetry (TG), differential scanning calorimetry (DSC)) and by ESR. The results are compared with thermoluminescence (TL) data to check if the same trapped electrons are observed by ESR and TL.

\section{Experimental}

ESR measurements were performed on a Radiopan SE/X-2547 and on a Bruker EMX spectrometer, working at $X$-band, with $100 \mathrm{kHz}$ magnetic modulation and a home-made high temperature ESR cavity of $\mathrm{TE}_{102}$-type, at a temperature range from room temperature up to $870 \mathrm{~K}$.

Samples of dripstone calcite were collected from the Tehuba part of the Black Cave (Jaskinia Czarna) at the Western Tatra Mountains, Poland, in the form of $20 \mathrm{~cm}$ long fragment of calcite layer covering a goaf. The samples were divided into smaller fragments, crushed, cleaned, grounded in a ceramic mortar and sieved with a final grain size lower than $0.063 \mathrm{~mm}$. The samples used in ESR experiments were annealed in the resonance cavity of the spectrometer in air or in nitrogen atmosphere. Some samples were $\gamma$-irradiated prior to ESR measurements with doses up to $300 \mathrm{~Gy}$.

DTA and DSC measurements were performed on a Perkin Elmer DSC 7 apparatus and the TG measurements on a Derivatograph Q-1500D.

\section{Results and discussion}

\subsection{ESR spectra}

ESR spectra of the dripstone calcite samples collected at different sites of the Black Cave consist of many ESR lines forming badly resolved spectra. Except the $\mathrm{Mn}^{2+}$-ion lines, typical of natural oxide impurities, the broad line with peak- 


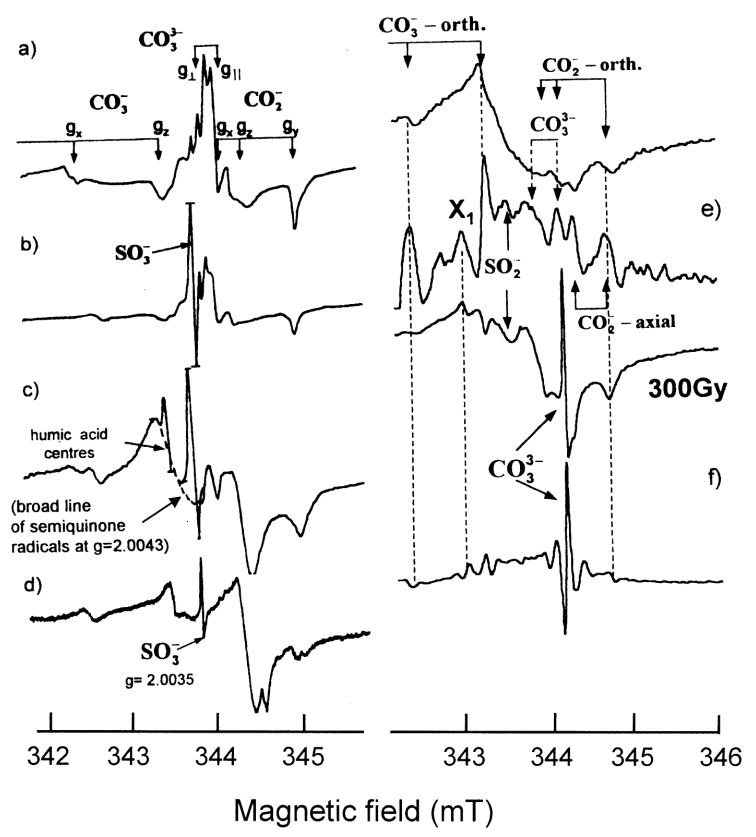

Fig. 1. Powder ESR spectra: (a) synthetic pure polycrystalline $\mathrm{CaCO}_{3}$ after $\gamma$-irradiation at room temperature $[1,8]$, (b) synthetic $\mathrm{CaCO}_{3}$ doped with $\mathrm{SO}_{3}^{-}$[7], (c) synthetic $\mathrm{CaCO}_{3}$ doped with humic acids [8], (d) calcite from stalactite of Akiyoshi cave (Japan) [1]; (e) our sample spectrum recorded at $9.642 \mathrm{GHz}$ and its enhanced resolution picture (in absorption mode) after FT procedures CREM, (f) our sample spectrum after $\gamma$-irradiation (dose $300 \mathrm{~Gy}$ ) and its enhanced resolution picture in absorption mode. The lines characteristic of some radicals are marked.

-to-peak linewidth $\Delta B_{\mathrm{pp}} \approx 0.9 \mathrm{mT}$ at $g=2.004$ is visible. The central part of the ESR spectrum, between the hyperfine $m_{I}= \pm 1 / 2 \mathrm{Mn}^{2+}$ lines, is shown in Fig. 1e, and the $\mathrm{Mn}^{2+}$ lines were used as markers for precise determination of the $g$-factors. The overlapped narrow lines indicate that various radical centres were formed by natural radiation during sample history. Identification of the centres directly from the room temperature ESR spectrum (Fig. 1e) is practically impossible except the low-field part of the spectrum (around $342 \mathrm{mT}$ ) where $\mathrm{CO}_{3}^{-}$radical lines are expected, and the high-field part (around $345 \mathrm{mT}$ ) where $\mathrm{CO}_{2}^{-}$lines can appear. Very helpful in an identification of paramagnetic centres are $g$-factors tabularised for various radical anions in natural and synthetic calcites [1] and the shape of ESR spectra from individual centres can be recognized in spectra of synthetic calcite and in intentionally doped crystalline calcite samples, as presented in Fig. 1a, b, c.

Except ESR lines from various types of carbonate anion radicals $\left(\mathrm{CO}_{3}^{-}\right.$, $\mathrm{CO}_{3}^{3-}, \mathrm{CO}_{2}^{-}$) being the intrinsic defects of $\mathrm{CaCO}_{3}$ lattice $[1,8]$, the signals from impurities related to paramagnetic defects can be observed. The position and 
shape of some of them can be recognized in Fig. 1b, c, i.e. an $\mathrm{SO}_{3}^{-}$isotropic line at $g=2.0035$ [7], and humic acids centres (mainly semiquinone-type radicals) lines: a narrow line at $g=2.0043\left(\Delta B_{\mathrm{pp}} \approx 0.6 \mathrm{mT}\right)$ superimposed on a very broad line $\Delta B_{\mathrm{pp}} \approx 6 \mathrm{mT}$ at $g \approx 2$ [8]. The $\mathrm{SO}_{3}^{-}$-line is visible also in the spectrum of natural calcite from stalactite of the Akiyoshi cave (Fig. 1d) and has been observed also in a synthetic "pure" crystalline calcite after $\gamma$-irradiation (dose 5 kGy) [8]. Moreover, an additional very sharp signal very often appears after $\gamma$-irradiation at $g=2.0001$. This is due to colour centres produced during grinding (accompanied by overheating) on the grain surfaces $[3,4]$.

A total number of unpaired electrons in the sample was determined by double integration of ESR spectrum with resulting paramagnetic centres density $9( \pm 4) \times 10^{13}$ centres/gram. This relatively low value of the spin concentration in the natural sample indicates that either the natural radiation in the Black Cave is not very intensive or the sample age is not high. In fact in our previous paper [14] we have determined the age of the samples from the Black Cave as 400-1400 kyears from ESR spectra and as 500-2100 years from ${ }^{230} \mathrm{Th} /{ }^{234} \mathrm{U}$ dating method, depending on the site in the cave. The small concentration of the spins indicates that the interaction between paramagnetic centres is rather small, except possible clusters. However, the apparent linewidth is larger than that in the single crystal spectra (Fig. 1a, b, c) as a result of a special distribution of the spectral parameters due to existing disorder.

A comparison of our sample spectrum (Fig. 1e) with the standard spectra (Fig. 1a, b, c) shows that an identification of the individual lines is still impossible by a simple comparison. To make it easier we have performed: (a) $\gamma$-irradiation which can induce or influence some centres, (b) enhancement of the spectral resolution using Fourier convolution-deconvolution procedure of computer resolution enhancement method (CREM), (c) annealing of the sample in air and in inert atmosphere which can simplified the spectrum.

Samples irradiated with $\gamma$-rays from ${ }^{60} \mathrm{Co}$ source display a significant change in the shape of ESR spectrum as shown in Fig. 1f. The spectrum is dominated by the lines from $\mathrm{CO}_{3}^{3-}$ species which, however, are not very stable and slowly disappear in time. But the spectrum of the irradiated sample allows one to determine the position and $g$-factors of the $\mathrm{CO}_{3}^{3-}$ radicals. Details of dependence of the ESR line amplitudes on the irradiation dose for samples of dripstone of the Black Cave can be found in the paper [14].

\subsection{Computer resolution enhancement method}

Computer resolution enhancement method is the splin-based deconvolution technique used for enhancing the resolution of overlapping ESR lines. Theoretical background of the method is described in the papers [15-17] and details of the algorithm can be found in [18]. Deconvolution of the filter (core) signal (which is assumed Gaussian or Lorentzian in the algorithm) from an experimental spectrum 
is performed by dividing the two signals in the Fourier domain. Subsequent Fourier transform gives a spectrum with effectively narrowed lines displaying artificially improved apparent peaks allowing an easy identification of the line positions. This method is especially effective in improvement of single crystal ESR spectra but it works also in the case when an initial spectrum is a powder-like spectrum containing peaks from anisotropic $g$-factors or hyperfine splittings. The algorithm works on absorption ESR spectra and resulting improved spectra are also in the absorption form. Carefully used the algorithm does not produce a deformation of the spectrum but delivers information on a number of lines in the spectrum, their position, and intensity ratio.

The spectra with enhanced resolution by the CREM method accompany the experimental spectra presented in Fig. 1e, f and Fig. 2. Great improvement in the resolution has been achieved allowing a precise determination of the line $g$-factors and discrimination between radical centres. Except the various carbonate radicals characteristic of $\mathrm{CaCO}_{3}$ matrix we have identified the $\mathrm{SO}_{2}^{-}$species. The $g$-factors for identified centresare summarized in the Table, where they are compared with

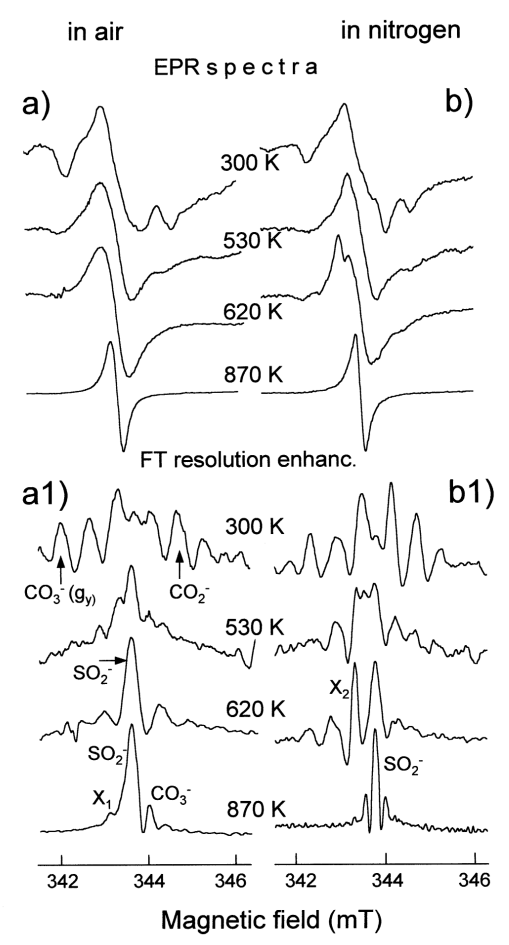

Fig. 2. Temperature evolution of the ESR spectra annealed in air (a) and in nitrogen (b) in the spectrometer cavity and they FT resolution enhancement pictures (a1 and b1). The highest temperature spectra are overdominated by signals from $\mathrm{SO}_{2}^{-}$ radicals. 
TABLE

EPR $g$-factors* of paramagnetic centres in natural calcite $\mathrm{CaCO}_{3}$.

\begin{tabular}{|c|c|c|c|c|}
\hline Centre & $g_{x}$ & $g_{y}$ & $g_{z}$ & Ref \\
\hline $\mathrm{CO}_{3}^{3-}$ & \multirow{2}{*}{\multicolumn{2}{|c|}{$\begin{array}{l}2.0032 \\
2.0031\end{array}$}} & 2.0014 & this paper \\
\hline & & & 2.0013 & {$[19]$} \\
\hline \multirow[t]{2}{*}{$\mathrm{CO}_{3}^{-}$} & 2.0120 & 2.0180 & 2.0056 & this paper \\
\hline & 2.0132 & 2.0194 & 2.0055 & {$[20]$} \\
\hline $\mathrm{CO}_{2}^{-}$ & 2.0032 & 1.9988 & 2.0014 & this paper \\
\hline orthorhombic & 2.0032 & 1.9971 & 2.0016 & {$[21]$} \\
\hline $\mathrm{CO}_{2}^{-}$ & & 1.9996 & 2.0025 & this paper \\
\hline axial & & 1.9994 & 2.0032 & {$[6]$} \\
\hline $\mathrm{SO}_{2}^{-}$ & & 2.0057 & & this paper \\
\hline isotropic & & 2.0053 & & {$[1,7]$} \\
\hline grinding & \multirow{2}{*}{\multicolumn{2}{|c|}{$\begin{array}{r}\text { not observed } \\
2.0001\end{array}$}} & & this paper \\
\hline signal & & & & {$[3,4]$} \\
\hline
\end{tabular}

${ }^{*}$ Errors: \pm 0.0003

literature data for the other cave deposits. Except ESR lines identified as shown in the Table, we have observed two additional lines marked as $X_{1}(g=2.0070)$ and $X_{2}(g=2.0078)$ in the spectra in Fig. 1e, f and Fig. 2a1, b1. Some indications on the origin of these lines have been found in changes of the spectra with temperature as described further. The spectrum contains also a broad line $\Delta B_{\mathrm{pp}} \approx 5 \mathrm{mT}$ at about $g=2$ which can be due to humic acid contaminations.

\subsection{Temperature effects in the ESR spectrum}

Thermal treatment of a sample during the ESR dating procedure can be crucial to the final results. A response of different radical centres on annealing temperature [1, 22, 23] and thermal stability [3] during isothermal annealing have been studied for various materials.

Annealing of our sample in the spectrometer cavity with a subsequent recording of the ESR spectrum produces visible changes depending on the temperature. Heating in air produces a different temperature dependence as compared to the variations observed in the nitrogen atmosphere. The temperature effects are not well recognized in the recorded ESR spectra (Fig. 2a, b), except the changes in the total spectral intensity, but the changes are clearly visible in their enhanced resolution pictures (Fig. 2a1, b1). Two characteristic effects can be recognized: (i) at highest temperatures the spectrum is dominated by the line from the $\mathrm{SO}_{2}^{-}$species; (ii) a new ESR line $X_{2}$ appears at high temperature in the nitrogen atmosphere only and grows in intensity with temperature. 


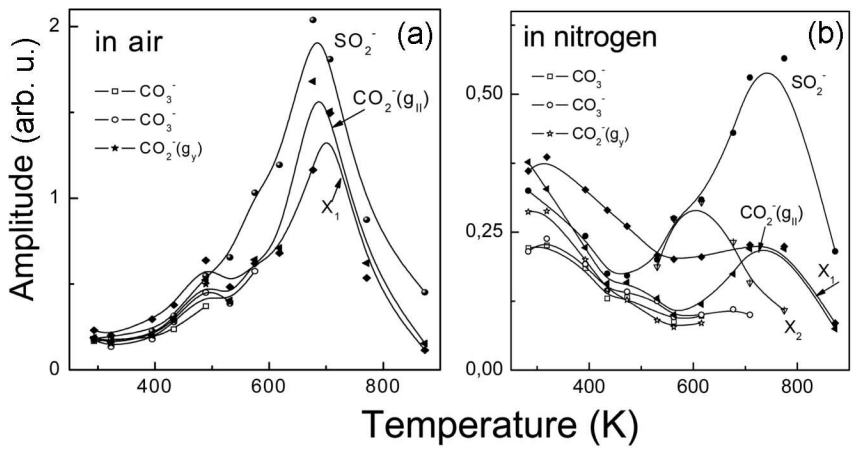

Fig. 3. Temperature dependence of the ESR line amplitudes for various anion radical centres in air (a) and nitrogen (b) atmosphere.

Amplitudes of the lines determined from absorption CREM spectra vary with temperature as shown in Fig. 3. The behaviour in the air and $\mathrm{N}_{2}$ is clearly different. Heating in the air produces a continuous increase in amplitude of all lines up to about $700 \mathrm{~K}$ with a small anomalous increase at about $490 \mathrm{~K}$. Amplitude variations in the nitrogen atmosphere are four times smaller and different in character - all amplitudes drop on heating except the lines from $\mathrm{SO}_{2}^{-}$and $\mathrm{CO}_{2}^{-}\left(g_{\|}\right.$-line) which maximize at about $760 \mathrm{~K}$. Moreover, a new line $X_{2}$ appears at $500 \mathrm{~K}$ with a maximum amplitude at about $600 \mathrm{~K}$.

The results indicate that the most stable are $\mathrm{SO}_{2}^{-}$radical ions producing the isotropic ESR line due to free reorientations. This line cannot be annealed out even at the highest temperatures.

Annealing of samples in the nitrogen atmosphere is not suitable prior to the dating procedure since it results in diminishing the total spectral intensity, whereas annealing in the air produces an increase in the total intensity accompanied, unfortunately, by the lost of resolution due to the line broadening.

The temperature behaviour of the $X_{1}$ line in the air is very similar to that of $\mathrm{SO}_{2}^{-}$and $\mathrm{CO}_{2}^{-}$lines. Considering this behaviour and the $g$-factor value of 2.0070, one can suggest that the line is produced by another $\mathrm{CO}_{2}^{-}$-centre similar to that observed in anhidrite $\left(\mathrm{CaSO}_{4}\right)$ which have $g_{y}=2.0082$ [23]. The line $X_{2}$ observed in a limited temperature range of $500-800 \mathrm{~K}$ in the $\mathrm{N}_{2}$-atmosphere can only be attributed, at least tentatively, to $\mathrm{NO}_{3}^{2-}$ centres having $g_{\perp}=2.0066$ and observed in calcite samples [24].

\subsection{Comparison of thermoluminescence and ESR results}

Thermoluminescence is the technique monitoring light emitted after thermal stimulation of materials containing trapped unpaired electrons or holes. These metastable centres are produced by natural radiation in minerals and their concentration depends on the total dose or radiation, i.e. on the time of the defects accumulation. The electrons or holes released from the traps by heating recombine 
emitting light. Intensity of the emitted light depends on the concentration of the electrons. Thus TL is used, similarly to ESR, as a method of dating [25]. TL light intensity is recorded while a sample is progressively heated mostly in vacuum or in an inert atmosphere. The variation of TL intensity vs. temperature is called the glow curve.

Figure 4 shows the distinct shapes of the glow curves, recorded during the dating procedure, for various materials. Two main peaks appear in all samples suggesting two main release and recombination mechanisms. They are marked as $B$ and $C$. The peak positions are shifted one another because different heating rates were used. It seems that peaks $B$ and $M$ have the same origin. The peak at about $550 \mathrm{~K}$ (marked as $M$ ) is generally used in the dating measurements.

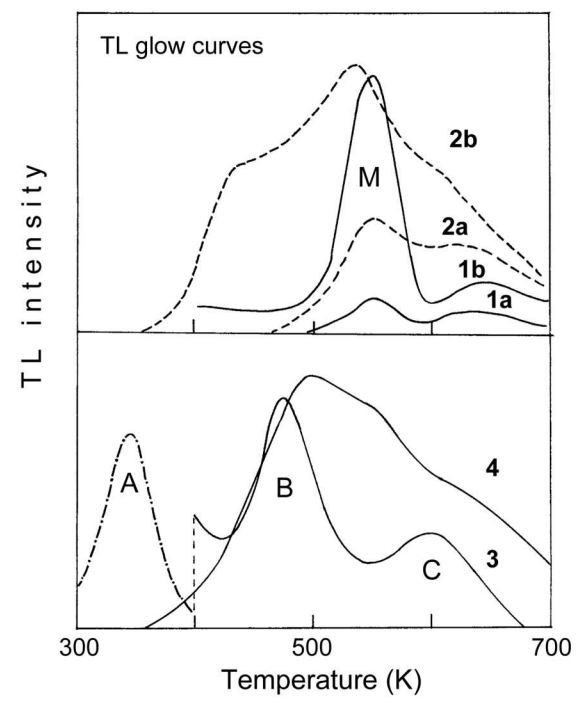

Fig. 4. Thermoluminescence glow curves for various materials used in TL dating: 1 - stalagmite calcite [26]: (a) natural sample, (b) $\gamma$-irradiated sample with a dose of 119 Gy; 2 - quaternary sediment sample QTL120B [27]: (a) natural sample, (b) $\gamma$-irradiated sample with a dose of $120 \mathrm{~Gy} ; 3$ - stalagmite sample [1]; 4 - middle neolitic age clay pot [28]. The peaks $A, B, C$, and $M$ are described in the text.

Natural calcite samples (curves 1 and 3 in Fig. 4) display generally TL peaks in temperature ranges (depending on the heating rate): $320-400 \mathrm{~K}$ (peak $A$ ), 470-570 K (peak $B$ ), and 600-670 K (peak $C$ ). If the thermally activated luminescence centres are paramagnetic one should observe a correlation between ESR and TL results and thus conclude about nature of the TL centres which is rare discussed during TL dating measurements.

The peak $A$ is observed only immediately after $\gamma$-irradiation of a sample and vanishes in hours. The same behaviour we observe for the ESR line of $\mathrm{CO}_{3}^{3-}$ centres which dominates in irradiated samples and has a very short lifetime. Thus 
we conclude that the peak $A$ is associated with migration of $\mathrm{CO}_{3}^{3-}$ electron centres. The peaks $M$ and $B$ seem to have the same origin and correlate with anomalies in ESR line amplitudes visible at about $500 \mathrm{~K}$ in Fig. 3. The peaks can be due to various carbonate radical centres although it has been strongly suggested that the peak $B$ is related to the $\mathrm{CO}_{2}^{-}$[1]. The high temperature peak $C$ is clearly due to the $\mathrm{SO}_{2}^{-}$centres which dominate in ESR spectra both in the air and especially in the nitrogen atmosphere.

\subsection{Thermal analysis}

Global thermal properties of our samples can be observed by thermal analysis methods. We have performed such measurements by TG, DTA, and DSC methods using a heating rate of $10 \mathrm{deg} / \mathrm{min}$, both for natural and for $\gamma$-irradiated samples. In DSC measurements a sample was heated up to $750 \mathrm{~K}$, then cooled down to room temperature and heated up again to observe a sample response after annealing during the first run. The thermal analysis results are shown in Fig. 5.

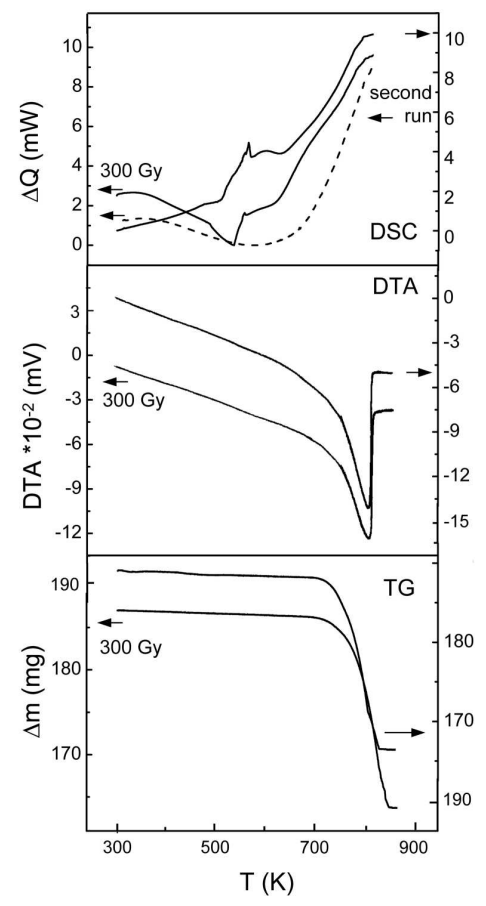

Fig. 5. Thermal analysis results for natural (right scale) and $\gamma$-irradiated sample (left scale) (a dose of $300 \mathrm{~Gy}$ ). The dotted line is a second run of the heating for the irradiated sample. The continuous decrease in the temperature in the DTA plots is an artefact due to the non-calibrated thermocouple.

It is well known that the calcite belongs to the group of volatile-bearing minerals which decompose with a single endothermic dissociation reaction liberating 
$\mathrm{CO}_{2}$ at high temperatures and formation of the oxide according to the reaction $\mathrm{CaCO}_{3}$ (solid) $\rightarrow \mathrm{CaO}$ (solid) $+\mathrm{CO}_{2}$ (gas) $[29,30]$. The decomposition of crystalline calcite appears at $934 \mathrm{~K}$ [29] and is observed as a lowering of the sample temperature in DTA and a loss of mass in TG measurements. In our sample the decomposition temperature determined from the endothermic peak in DTA and from $\mathrm{TG}$ is about $820 \mathrm{~K}$ which is much lower than that for the crystalline calcium carbonate suggesting rather a metastable amorphous structure. Dripstone calcite is rather an impure material containing many inorganic and organic impurities as suggested by the ESR results. Moreover, most of natural calcites contain absorbed water which is the second most volatile component of the compounds. Dehydratation of calcites is generally observed as exothermic effects in DSC curves and appears until about $530 \mathrm{~K}$. This is accompanied by a subsequent crystallization of dissolved calcite [31] which is considered as the main destructive mechanism in decay of buildings and monuments built with the limestone.

DSC curves of our natural and irradiated samples show the heat flow typical of amorphous materials with a continuous exothermic effect. The natural sample curve shows an additional exothermic effect in a temperature range of 500-620 K which can be due to evaporation of water and crystallization or more probable a decomposition of organic components. These effects do not affect the TG curve indicating a very small change in the mass of the sample below $700 \mathrm{~K}$. At the second run of the heating the exothermic effect is strongly reduced below of about $700 \mathrm{~K}$ indicating the annealing effect of the sample. The DSC curve of this run is very similar to that presented as a dashed line for irradiated sample in Fig. 5. The $\gamma$-irradiation with a dose of 300 Gy also leads to annealing the sample with a continuous endothermic effect below $500 \mathrm{~K}$.

\section{Conclusions}

The calcite collected from dripstone layer of the Black Cave of the Tatra Mountains is an amorphous material (as shown by the thermal analysis data) containing inorganic (sulphur salts, nitrate salts) and organic (humus) impurities (as identified in ESR spectra). A large amount of $\mathrm{SO}_{2}^{-}$anions dominating a high temperature ESR spectrum suggests a strong contamination by sulphur containing compounds which can origin from pyrite $\left(\mathrm{FeS}_{2}\right)$ rich rocks (dark limestones) surrounding the Black Cave.

ESR spectra as recorded are not suitable for a detailed discussion. Additional analysis of computer enhanced resolution spectra, influence of temperature and ionising irradiation are needed for identification of paramagnetic centres. Such analysis shows that the central part of ESR spectrum, which very often apparently looks like a single line, is in fact composed from many ESR lines of various radical centres. Such line, in our opinion, is not suitable for the ESR dating when an additional irradiation is used for determination of the total dose of natural 
radiation, although this multicomponent line is commonly used in the dating procedures $[4,32]$.

Instead of this we strongly suggest that the best lines for the ESR dating are the outer lines of the spectrum being well separated from the other lines, i.e. $\mathrm{CO}_{2}^{-}$-line with $g_{y}=1.9980(3)$, and $\mathrm{CO}_{3}^{-}$-lines with $g_{x}=2.0125(5)$ and $g_{y}=2.0185(5)$ as it was also suggested in [33].

Annealing of the samples at moderated temperatures does not improve the spectral resolution and not disturb the relative amplitudes of the individual lines up to about $500 \mathrm{~K}$.

Electron spin resonance and thermoluminescence methods, which are complementary in geological dating, give consistent results in the interpretation of the thermal effects in ESR spectra and TL glow curves indicating that the same centres are involved in dating procedures of the both methods.

\section{Acknowledgment}

This work was supported by the Ministry of Scientific Research and Information Technology under grant No. 3 P04D 03022.

\section{References}

[1] M. Ikeya, New Applications of Electron Spin Resonance-Dating, Dosimetry and Microscopy, World Sci., Singapore 1993.

[2] M. Ikeya, Adv. ESR Appl. 18, 321 (2002).

[3] D.F. Regulla, A. Wieser, H.Y. Göksu, Nucl. Tracks 10, 825 (1985).

[4] R. Grün, Nucl. Tracks Radiat. Meas. 18, 143 (1991).

[5] S.A. Marshall, A.R. Reinbaerg, R.A. Serway, J.A. Hodges, Mol. Phys. 8, 225 (1964).

[6] J.A. McMillan, S.A. Marshall, J. Chem. Phys. 48, 467 (1968).

[7] A. Kai, T. Miki, Jpn. J. Appl. Phys. 30, 1109 (1991) and Radiat. Phys. Chem. 40, 469 (1992).

[8] P. DeCanniere, Th. Joppart, R. Debuyst, F. Dejehet, D. Apers, Nucl. Tracks 10, 853 (1985).

[9] K. Meguro, M. Ikeya, Jpn. J. Appl. Phys. 32, 3540 (1993).

[10] D.C. Ford, P.W. Williams, Karst Geomorphology and Hydrology, Chapman and Hall, Boston 1989.

[11] R.J. Reeder, Carbonates-Mineralogy and Chemistry, Mineral. Soc. Amer., Washington 1990.

[12] P. Kowalik, Soli Protection, PWN, Warszawa 2001 (in Polish).

[13] E. Mysliwska, Laboratory Analysis of Soil, PWN, Warszawa 2001 (in Polish).

[14] M. Wencka, R. Krzyminiewski, Appl. Magn. Res. 26, 561 (2004).

[15] A. Hedberg, A. Ehrenfest, J. Chem. Phys. 48, 4822 (1968). 
[16] A. Koper, R. Krzyminiewski, Acta Magnetica 2, 3 (1985).

[17] V.K. Madisetti, D.B. Williams, The Digital Signal Processing Handbook, CRS Press, New York 1998.

[18] R. Krzyminiewski, R.M. Kowalczyk, A. Bielewicz-Mordalska, Z. Pajak, P. Czarnecki, J. Mol. Struct. 471, 234 (1998).

[19] R.A. Servay, S.A. Marshall, J. Chem. Phys. 46, 1949 (1967).

[20] R.A. Servay, S.A. Marshall, J. Chem. Phys. 47, 868 (1967).

[21] A. Rossi, G. Poupeau, Appl. Radiat. Isot. 40, 1133 (1989).

[22] O.G. Duliu, Appl. Radiat. Isot. 52, 1385 (2000).

[23] A.J. Kailath, T.K.G. Rao, R.P. Dhir, K.S.V. Nambi, V.D. Gogte, A.K. Singhvi, Radiat. Meas. 32, 371 (2000).

[24] R.S. Eachus, M.C.R. Symons, J. Chem. Soc. (A) 45, 790 (1968).

[25] M.J. Aitken, Thermoluminescence Dating, Academic Press, London 1985.

[26] Quaternary TL Surveys. http://www.users.globalnet.co.uk/ qtls/calcite.htm.

[27] A.G. Wintle, in: Quaternary Dating Methods. A User Guide, Eds. P.L. Smart, P.D. Frances, Quaternary Research Association, Cambridge 1991.

[28] T. Berger, Thermoluminescence Dating, http://www.lumenarchaeology.com/lfs/technology.htm.

[29] B.V. Lvov, Thermochim. Acta 386, 1 (2002).

[30] D.T. Beruto, A.W. Searcy, M.G. Kim, Thermochim. Acta 424, 99 (2004).

[31] N. Koga, Y. Nakagoe, H. Tanaka, Thermochim. Acta 318, 239 (1998).

[32] P.I. Smart, in: Quaternary Dating Methods. A User Guide, Eds. P.L. Smart, P.D. Frances, Quaternary Research Association, Cambridge 1991.

[33] M. Ikeya, T. Miki, J. Speleol. Soc. Jpn. 9, 36 (1984). 